\title{
Dynamic Ion Pairs in the Adsorption of Isolated Water Molecules on Alkaline-Earth Oxide (001) Surfaces
}

\author{
Javier Carrasco, ${ }^{1}$ Francesc Illas, ${ }^{1}$ and Nuria Lopez ${ }^{2, *}$ \\ ${ }^{1}$ Departament de Química Física \& IRQTC, Universitat de Barcelona \& PCB, C/Martí i Franquès 1, 08028 Barcelona, Spain \\ ${ }^{2}$ Institute of Chemical Research of Catalonia, ICIQ, Avgda. Països Catalans 16, 43007 Tarragona, Spain
}

(Received 27 June 2007; published 3 January 2008)

\begin{abstract}
Low coverage water adsorption on alkaline-earth oxides is studied by first principles methods and ab initio molecular dynamic simulations. $\mathrm{On} \mathrm{CaO}$ and $\mathrm{BaO}(001)$, water dissociation is thermodynamically favored in contrast with the molecular adsorption found for $\mathrm{MgO}(001)$. On $\mathrm{CaO}(001)$, the barrier for water splitting is very small leading to a tight ion pair $\mathrm{H}-\mathrm{OH}$ that exhibits a dynamic character with the hydroxyl group able to visit four equivalent adsorption sites while remaining attached to the proton. In contrast, ion pair separation is endothermic by $0.8 \mathrm{eV}$. These results are common to other basic surfaces such as $\mathrm{BaO}(001)$ and have important implications in the chemistry of partially hydroxylated oxide surfaces.
\end{abstract}

Water interaction with solid surfaces remains at the core of several fields such as corrosion, electrochemistry, geochemistry, and biocompatible materials [1]. The interfacial water structure continues to be an issue of intense interest because gaining atomic level understanding of water-solid interactions constitutes a real challenge. The problem arises from the presence of multiple weak interactions and of the complex, almost isoenergetic, structures present under these conditions [2]. Recent reviews have extensively covered the large volume of literature published to date $[1,3,4]$ although quantitative structural determinations are scarce [5-7]. Under ambient conditions, many oxides are partially covered by $\mathrm{H}_{2} \mathrm{O}$ molecules and/or hydroxyl groups [8]. For instance, a water monolayer on defect free $\mathrm{MgO}(001)$ shows partial water dissociation [9], while molecular adsorption was suggested from Density Functional Theory (DFT) calculations on $\mathrm{CaO}(001)$ [10] and ion pairs have been described for stepped $\mathrm{MgO}$ [11]. In contrast, a complex dynamic behavior has been described for a water monolayer on $\mathrm{ZnO}(10-10)$ [12]. In a naïve way, the energy contributions to water adsorption at high coverage can be split in two parts: (i) the isolated water molecule-surface interaction, and (ii) water-water interaction. The latter promotes water dissociation and hydroxyl formation, while the role of the substrate, i.e., inducing water dissociation or molecular adsorption, is less clear [13]. In fact, most of the studies of water adsorption on metal oxides have focused on the substrate ability to induce water dissociation $[3,4,14]$. The aim of the present Letter is to investigate the strength of the isolated water-oxide interaction, to identify the contributions that govern the dissociated or molecular adsorption, and to understand their dynamic behavior for simple alkaline-earth oxide surfaces. In the following, we present evidence for the different behavior of an isolated water molecule on $\mathrm{MgO}$ and $\mathrm{CaO}$ or $\mathrm{BaO}(001)$.

In order to study the properties of isolated water molecules on oxide surfaces, we have performed a series of DFT calculations on slabs representing $\mathrm{MgO}$ and $\mathrm{CaO}(001)$ surfaces complemented by ab initio molecular dynamics (AIMD) simulations. Key calculations have also been performed for $\mathrm{BaO}(001)$. For systems showing multiple lowlying minima separated by small energy barriers, the use of the AIMD uncovers the most relevant structures present in the corresponding Potential Energy Surface, PES, and can be employed as a conformational explorer. With this information, it is then possible to perform more accurate static calculations to obtain energies for all the possible processes, even those not seen in the AIMD simulations due either to too short simulation times or to too high reaction barriers. To evaluate the energy, the PW91 exchange-correlation potential has been used [15]. The effect of core electrons in the valence density is taken into account by means of the Projector Augmented Wave (PAW) method [16] while the valence density is expanded in a plane-wave basis set with a cutoff energy of $415 \mathrm{eV}$.

In the static calculations, a single water molecule is adsorbed on 4 atomic layers metal-oxide slabs cut along the (001) direction, with the two oxide bottom layers fixed to their bulk PW91-optimal positions $\left(a_{\mathrm{MgO}}=4.238 \AA\right.$, $a_{\mathrm{CaO}}=4.843 \AA$, and $a_{\mathrm{BaO}}=5.610 \AA$ ). Repeated slabs are interleaved by $14 \AA$ vacuum. The $4 \sqrt{ } 2 \times 4 \sqrt{ } 2$ surface supercell is employed, equivalent to $1 / 16 \mathrm{ML}$ coverage with a minimum water-water distance of $4 \sqrt{ } 2 a_{0}$. A $2 \times$ $2 \times 1$ Monkhorst-Pack grid [17] has been used for $k$-point sampling. The transition state (TS) structures for relevant processes have been located by the climbing image nudged elastic band (CI-NEB) [18] and characterized by vibrational analysis. For AIMD calculations, the size of the supercells has been reduced to $3 \sqrt{ } 2 \times 3 \sqrt{ } 2$ and 2 layers, with $10 \AA$ vacuum and the oxide bottom layer was frozen at its bulk optimal geometry. The AIMD calculations were performed under the Born-Oppenheimer approximation where the free energy (at the $\Gamma$-point only) can be evaluated using conventional DFT methods. The canonical ensemble (NVT) at a prefixed temperature, fixed through a Nosé thermostat [19], has been employed. For the sys- 
tems of interest, the accuracy of each electronic minimization is crucial for a correct time evolution and therefore it has been increased to $10^{-7} \mathrm{eV}$. To keep the system stable at the temperatures considered here $(100$ and $300 \mathrm{~K})$, the Nosé mass parameter was set equal to $24.3 \times 10^{-27}$ a.u. This set up guarantees a good energy conservation measured by a drift of the expectation value of the extended system (ions + electrons + Nosé thermostat) smaller than $0.001 \mathrm{eV} / \mathrm{ps}$ per unit cell. Initial equilibration steps were performed over 1-2 ps, while production runs were 1020 ps long. All simulations have been performed using the VASP code [20].

The equilibrium geometry and adsorption energy of water molecule on $\mathrm{BaO}, \mathrm{CaO}$, and $\mathrm{MgO}(100)$ are reported in Fig. 1. Although we identified different $\mathrm{H}_{2} \mathrm{O}$ adsorption structures, only the low-energy ones are discussed here (A1). Both O-down (only $1 \mathrm{H}$ pointing to the surface) and $\mathrm{H}$-down (two $\mathrm{H}$ atoms pointing to the surface) structures have been explored for $\mathrm{CaO}(001)$. The minimum energy corresponds to a symmetric configuration with both $\mathrm{H}$ atoms pointing towards $\mathrm{O}$ surface atoms; the adsorption energy is $-0.80 \mathrm{eV}$ and the corresponding frequencies, 2728, 2666, and $1629 \mathrm{~cm}^{-1}$. An equivalent structure is retrieved for $\mathrm{BaO}(001)$, the binding energy being $-0.88 \mathrm{eV}$. On $\mathrm{MgO}$, the $\mathrm{O}$ atom of water is located

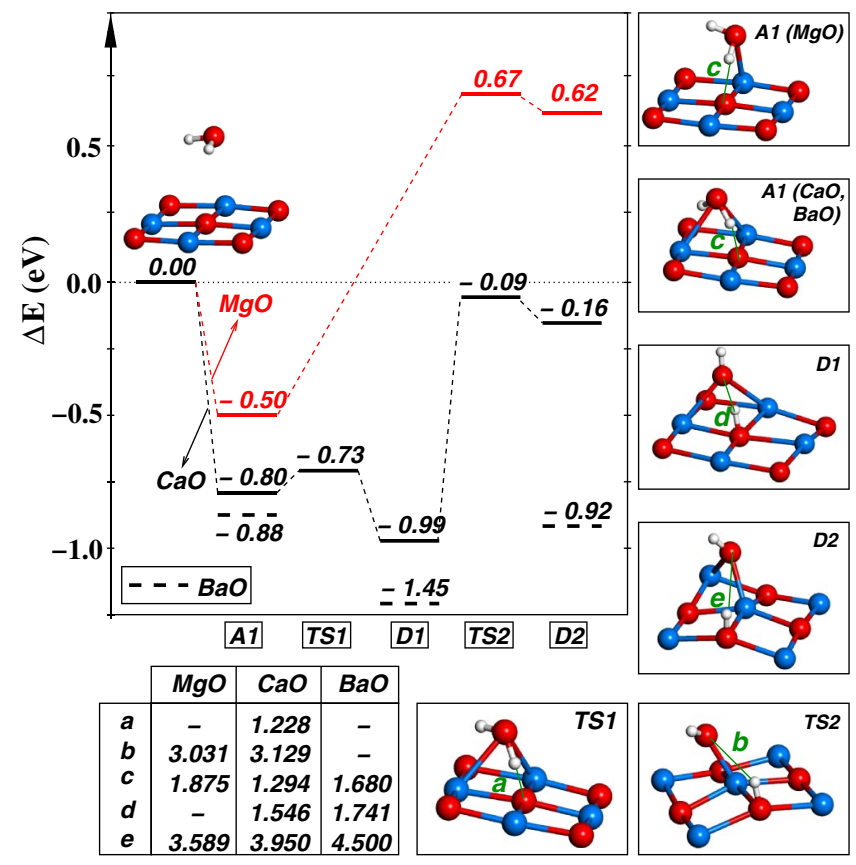

FIG. 1 (color online). Reaction energy profiles (in $\mathrm{eV}$ ) and atomic structures for isolated water dissociation on $\mathrm{MgO}, \mathrm{CaO}$, and $\mathrm{BaO}(001)$ from molecular to dissociated ion pairs for the minimum energy pathway. Insets show the corresponding geometry sketches for minima (A1, D1, and D2) and transition states (TS1 and TS2). The chart in the lower-left corner summarizes the targeted O-H distances (in $\AA$ ) pointed out in the insets. Small white spheres stand for $\mathrm{H}$ atoms, while red (light) and blue (dark) for $\mathrm{O}$ and cations, respectively. on top of a $\mathrm{Mg}^{2+}$, while $\mathrm{H}$ atoms are asymmetrically oriented with $1 \mathrm{H}$ pointing to the oxide surface $\left(d_{\mathrm{O}-\mathrm{H}}=\right.$ $1.027 \AA$ and $0.972 \AA$ ). The binding energy is $-0.50 \mathrm{eV}$, in excellent agreement with experimental data $(-0.52 \pm$ $0.10 \mathrm{eV})$ [21] and previous theoretical estimates [22]. Adsorbed $\mathrm{H}_{2} \mathrm{O}$ vibrational frequencies are 3746, 2767, and $1608 \mathrm{~cm}^{-1}$. The larger basicity of the $\mathrm{CaO}$ and $\mathrm{BaO}(001)$ surfaces [23] is behind the change in the molecular configuration from $\mathrm{O}-$ to $\mathrm{H}$-down on going from $\mathrm{MgO}$ to $\mathrm{CaO}$ or $\mathrm{BaO}$.

Water dissociation and separation (D2 structures in Fig. 1) on $\mathrm{CaO} / \mathrm{BaO}$ is exothermic by $0.16 / 0.92 \mathrm{eV}$ and endothermic for $\mathrm{MgO}(001)$ by $0.62 \mathrm{eV}$ with respect to the gas-phase molecule and the surface. The total energy can be seen as the energy for the proton and the hydroxyl group interacting mainly with the surface minus the energy needed to break the $\mathrm{H}-\mathrm{OH}$ bond. The latter is a constant, and, therefore, the energetics for $\mathrm{H}_{2} \mathrm{O}$ dissociation is governed by the proton-anion and hydroxyl-cation interaction. The larger basicity of $\mathrm{CaO}(\mathrm{BaO})$ with respect $\mathrm{MgO}$ is again behind the exothermicity for the reaction [24]. As observed in the inset of the D2 state in Fig. 1, a strong relaxation of the surface cations in the vicinity of adsorbed $\mathrm{OH} / \mathrm{H}$ species exists. In particular for $\mathrm{MgO}(\mathrm{CaO})$, an outwards relaxation of about $0.40(0.37) \AA$ for the first layer with respect to the clean slab is observed, while this effect is substantially mitigated in $\mathrm{BaO}(001), 0.06 \AA$.

The AIMD simulations for $\mathrm{CaO}(001)$ starting from an adsorbed molecular state, at 100 and $300 \mathrm{~K}$, show a con-

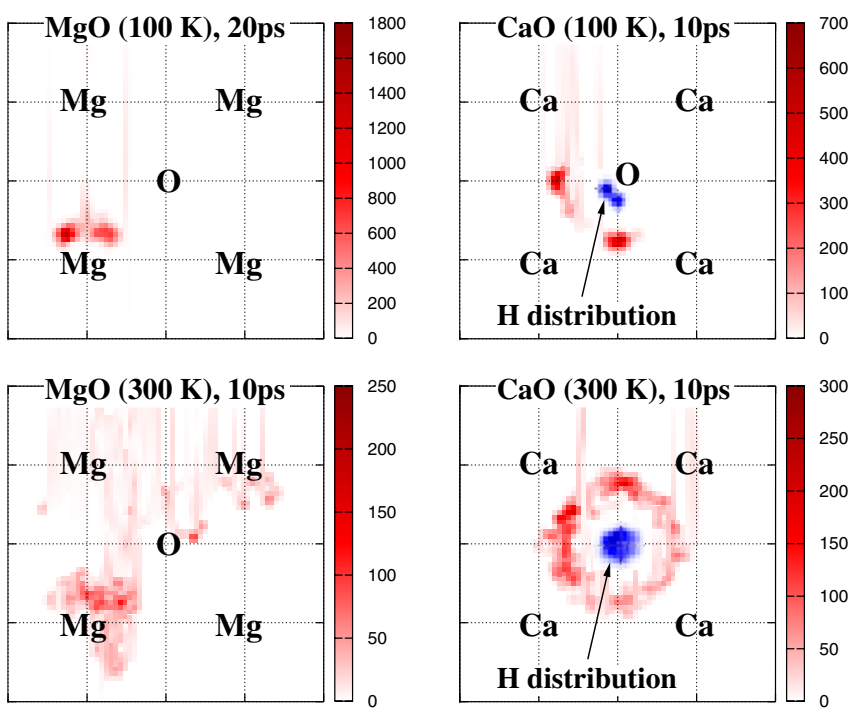

FIG. 2 (color online). Projection on the $\mathrm{MgO}$ and $\mathrm{CaO}$ (001) surface of the position of the oxygen atom corresponding to the $\mathrm{H}_{2} \mathrm{O}$ (MgO substrate) or $\mathrm{OH}_{\text {ads }}$ (CaO substrate) species from AIMD simulations at two different temperatures (100 and $300 \mathrm{~K}$ ) and simulation times (10 and $20 \mathrm{ps}$ ). Degraded color scale (dark to light) on the right indicates the number of configurations for a given discrete region of the surface plane. In the case of $\mathrm{CaO}$, also $\mathrm{H}_{\mathrm{ads}}$ is shown. 
figuration corresponding to the dissociated water structure D1, Fig. 1. The $\mathrm{OH}_{\text {ads }}$ group sits on bridge between two adjacent $\mathrm{Ca}^{2+}$, and $\mathrm{H}_{\text {ads }}$ is attached to the nearest surface anion. Hence, D1 configurations can be described as a tight ion pair in the sense that an attractive interaction between $\mathrm{OH}_{\mathrm{ads}}$ and $\mathrm{H}_{\mathrm{ads}}$ subsystems exists that holds the pair together. In fact, the ion pair keeps the entity along the total simulation times employed. In addition, AIMD runs reveal that, even at temperatures as low as $100 \mathrm{~K}$, the $\mathrm{OH}_{\text {ads }}$ group shows a very high mobility around the pinned $\mathrm{H}_{\text {ads }}$ (Fig. 2). When oscillating in D1 configurations, the shortest and longest $d_{\mathrm{O}-\mathrm{H}}$ in the $\mathrm{OH}_{\text {ads }}$ group are 1.04 and $0.91 \AA$; between $\mathrm{OH}_{\mathrm{ads}}$ and $\mathrm{H}_{\mathrm{ads}}$ groups, these distances are 1.04 to $1.75 \AA$. The same behavior is obtained for $\mathrm{BaO}(001)$ where also a dynamic ion pair is formed (not shown in the figures). In this case, the $\mathrm{OH}_{\mathrm{ads}}-\mathrm{H}_{\mathrm{ads}}$ distance fluctuates around a mean value, $\sim 1.6 \AA$.

To accurately evaluate the size of the barriers for $\mathrm{H}_{2} \mathrm{O}$ dissociation and $\mathrm{OH}_{\mathrm{ads}}-\mathrm{H}_{\mathrm{ads}}$ separation on $\mathrm{CaO}(001)$, we now return to the static CI-NEB calculations. Several conceivable paths were investigated but only the less energetically demanding is shown in Fig. 1. As indicated, the molecular A1 state is adsorbed by $-0.80 \mathrm{eV}$. The system can evolve through a small barrier, $0.07 \mathrm{eV}$, to reach the tight ion pair D1 state, which is more stable than the molecular state by $0.2 \mathrm{eV}$. At the TS leading to the ion pair, the interatomic distance between the $\mathrm{OH}_{\text {ads }}$ group oxygen atom and the pinned $\mathrm{H}_{\mathrm{ads}}$ is quite small, 1.228 A and the imaginary frequency is $650 \mathrm{~cm}^{-1}$. The split of the ion pair leading to separated $\mathrm{OH}_{\text {ads }}$ and $\mathrm{H}_{\mathrm{ads}}$ groups (D1 $\rightarrow$ D2) is endothermic by $0.83 \mathrm{eV}$ and hindered by a barrier of $0.90 \mathrm{eV}$. This high barrier explains why only D1 configurations are observed in the AIMD simulations (Fig. 2). In the D2 configuration, the $\mathrm{OH}_{\text {ads }}$ group bridges as well two $\mathrm{Ca}^{2+}$ ions, but it is noticeably tilted with respect to D1 configuration, and the distance between $\mathrm{OH}_{\mathrm{ads}}$ and $\mathrm{H}_{\mathrm{ads}}$ groups is considerable larger, $3.950 \AA$. At the TS, the $\mathrm{H}_{\text {ads }}-\mathrm{OH}_{\text {ads }}$ distance is $3.129 \AA$. Therefore, although water dissociation on $\mathrm{CaO}(001)$ is a relatively low-temperature process, it leads to the formation of tight ion pairs which are effortless to form but hard to dissociate.

These observations are in good agreement with the experimental description for water adsorption on $\mathrm{CaO}(001)$ where dissociation is observed even at low water coverage (i.e., $p=10^{-10}$ Torr) [25]. Moreover, the tight ion pair formed is dynamic as clearly shown by the AIMD simulations; the calculated barrier for the $\mathrm{OH}_{\text {ads }}$ movement around the pinned $\mathrm{H}_{\mathrm{ads}}$ is only $0.05 \mathrm{eV}$, and thus it is the fastest of all the studied processes. This behavior is similar for $\mathrm{BaO}(001)$ where the dynamic ion pair is adsorbed by $-1.45 \mathrm{eV}$ (with respect to gas-phase water) and its dissociation is endothermic by $0.53 \mathrm{eV}$ while the rotation barrier of $\mathrm{OH}_{\mathrm{ads}}$ around $\mathrm{H}_{\mathrm{ads}}$ is $0.10 \mathrm{eV}$.

In contrast, in the AIMD runs performed for $\mathrm{MgO}$, no dissociated water configuration was observed in agreement with previous investigations [13]. At low temperatures, $100 \mathrm{~K}$, the molecular structure A1 is recovered with only small oscillations, Fig. 2. At room temperature, $300 \mathrm{~K}$, isolated water molecules diffuse randomly on the surface. Such a behavior is consistent with the small adsorption energy found and the expected small diffusion energy. In fact, $\mathrm{H}_{2} \mathrm{O}$ dissociation on $\mathrm{MgO}(001)$ from the molecular adsorbed state (A1 $\rightarrow \mathrm{D} 2$ process) is endothermic by $1.12 \mathrm{eV}$, the corresponding barrier being $1.17 \mathrm{eV}$. At the TS, the $\mathrm{H}_{\mathrm{ads}}-\mathrm{OH}_{\text {ads }}$ groups are far from each other, $3.031 \AA$. If the water TS1 structure from the $\mathrm{CaO}$ system for the $\mathrm{A} 1 \rightarrow \mathrm{D} 1$ process is considered for $\mathrm{MgO}$, the $\mathrm{H}_{\mathrm{ads}}-\mathrm{OH}_{\mathrm{ads}}$ distance would be of $\sim 1 \AA$, indicating that in $\mathrm{MgO}$ this structure is not likely to be a transition state. The large barrier found explains why the $\mathrm{A} 1 \rightarrow \mathrm{D} 2$ process is not seen in the AIMD simulations. Thus, regular surface sites are inactive towards $\mathrm{H}_{2} \mathrm{O}$ splitting, but other sites like linear defects that have been proposed as active sites [14] are accessible at room temperature when diffusion is a likely event as seen in the AIMD simulations at $300 \mathrm{~K}$. However, when comparing the structures of the TS for dissociation and separation in the $\mathrm{MgO}$ and $\mathrm{CaO}$, we observe that the barrier for ion pair dissociation (D1 $\rightarrow$ D2) for $\mathrm{CaO}$ is almost as large as the barrier for molecular dissociation $(\mathrm{A} 1 \rightarrow \mathrm{D} 2)$ on $\mathrm{MgO}(0.9$ vs $1.1 \mathrm{eV})$.

Finally, it is important to notice that in isolated water adsorption, the hydroxyl group is always the diffusing object; the barriers for $\mathrm{H}_{\text {ads }}$ diffusion in the same conditions are more than $0.5 \mathrm{eV}$ higher (alternative D1 $\rightarrow$ D2 paths). This is in contrast with the behavior at high water coverages where proton diffusion through Grotthus mechanism would be favored. Therefore, although protons are smaller than hydroxyl groups, the latter are more prone to diffusion due to better coordination capabilities of $\mathrm{OH}_{\text {ads }}$. Same phenomena had been described for small metal aggregates [26].

Summarizing, water molecules on regular $\mathrm{MgO}(001)$ surfaces maintain their molecular character. Dissociation at room temperatures is not observed due to the large barriers involved in the single step formation and separation of the proton-hydroxyl structure. This is due to the short distances between the positive and negative ions in the crystal that lead to a poor basicity and a too short $\mathrm{H}_{\mathrm{ads}}-\mathrm{OH}_{\mathrm{ads}}$ distance at the likely transition state for dissociation. For $\mathrm{CaO}$ and $\mathrm{BaO}$, dissociated structures are thermodynamically preferred. These are better described as dynamic ion pairs since the adsorbed hydroxyl group can freely rotate around the pinned proton. However, the strength of the ion pair is such that its separation is hindered by large barriers. The dynamic ion pair is tighter and rotation is faster in the $\mathrm{CaO}$ system than for $\mathrm{BaO}$. The formation of tight ionic pairs on the oxide surface might have consequences in several surface properties. For instance, dynamic ion pairs would show large radial extensions in Scanning Probe Microscopy (SPM) measurements 
due to the dynamic occupation of a radius of $2^{*} d_{\mathrm{CaO}}$. Such behavior could be used as a measure of the active sites spatial extent; in fact, some SPM experiments have indicated a large extension of defects [27]. In addition, present results indicate that isolated hydroxyl and proton groups on the surface might be a too simplified model when dealing with low-hydrated oxide surfaces since the formation of ion pairs is energetically favored. Care must be taken in the simulations of the chemical properties of the partially water covered oxide surfaces to include water ion pairs that might strongly perturb the acid or base characteristics of the surface due to cooperative effects. Finally, isolated water dissociation on regular $\mathrm{CaO}(001)$ (or $\mathrm{BaO}(001)$ ) will further enhance the hydroxylation of the surface.

The authors thank Professors G. Pacchioni and A. Michaelides for critically reading the manuscript. Financial support comes from MEC (Nos. CSD2006-003, CTQ2006-00464BQU, CTQ2005-08459-CO2-01, and UNBA05-33-001), GenCat (No. 2005SGR-00697, 2005 PEIR 0051/69 and DU granted to F. I. and N. L.). We are indebted to BSC for computing time.

*Corresponding author: nlopez@iciq.es

[1] P. A. Thiel and T. E. Madey, Surf. Sci. Rep. 7, 211 (1987).

[2] J. Cerdá, A. Michaelides, and M.-L. Bocquet et al., Phys. Rev. Lett. 93, 116101 (2004).

[3] M. A. Henderson, Surf. Sci. Rep. 46, 1 (2002).

[4] A. Verdaguer, G. M. Sacha, H. Bluhm, and M. Salmeron, Chem. Rev. 106, 1478 (2006).

[5] G. Held and D. Menzel, Surf. Sci. 316, 92 (1994).

[6] A. Glebov, A. P. Graham, A. Menzel, and J.P. Toennies, J. Chem. Phys. 112, 11011 (2000).

[7] J.P. Toennies, F. Traeger, J. Vogt, and H. Weiss, J. Chem. Phys. 120, 11347 (2004).

[8] Z. Łodziana, N.-Y. Topsøe, and J. K. Nørskov, Nat. Mater. 3, 289 (2004).
[9] L. Giordano, J. Goniakowski, and J. Suzanne, Phys. Rev. Lett. 81, 1271 (1998).

[10] N. H. de Leeuw, J. A. Purton, and S. C. Parker et al., Surf. Sci. 452, 9 (2000).

[11] C. Chizallet, G. Costentin, and M. Che, et al., J. Phys. Chem. B 110, 15878 (2006).

[12] O. Dulub, B. Meyer, and U. Diebold, Phys. Rev. Lett. 95, 136101 (2005).

[13] W. Langel and M. Parrinello, J. Chem. Phys. 103, 3240 (1995).

[14] G.E. Brown, V.E. Hendrich, and W.H. Casey et al., Chem. Rev. 99, 77 (1999).

[15] J. P. Perdew and Y. Wang, Phys. Rev. B 45, 13244 (1992); J. P. Perdew, J. A. Chevary, and S. H. Vosko et al., Phys. Rev. B 46, 6671 (1992).

[16] G. Kresse and D. Joubert, Phys. Rev. B 59, 1758 (1999).

[17] H. J. Monkhorst and J. D. Pack, Phys. Rev. B 13, 5188 (1976).

[18] G. Henkelman, B. P. Uberuaga, and H. Jónsson, J. Chem. Phys. 113, 9901 (2000).

[19] S. Nosé, J. Chem. Phys. 81, 511 (1984); S. Nosé, Prog. Theor. Phys. Suppl. 103, 1 (1991).

[20] G. Kresse and J. Hafner, Phys. Rev. B 47, 558 (1993); G. Kresse and J. Furthmüller, Phys. Rev. B 54, 11169 (1996).

[21] D. Ferry, S. Picaud, and P. N. M. Hoang et al., Surf. Sci. 409, 101 (1998).

[22] F. Finocchi and J. Goniakowski, Phys. Rev. B 64, 125426 (2001).

[23] G. Pacchioni, J. M. Ricart, and F. Illas, J. Am. Chem. Soc. 116, 10152 (1994).

[24] J. Goniakowski, S. Bouette-Russo, and C. Noguera, Surf. Sci. 284, 315 (1993);

[25] P. Liu, T. Kendelewicz, and G. E. Brown, Jr et al., Surf. Sci. 416, 326 (1998); M. J. Iedema, N. Kizhakevariam, and J. P. Cowin, J. Phys. Chem. B 102, 693 (1998).

[26] V. Musolino, A. Selloni, and R. Car, Phys. Rev. Lett. 83, 3242 (1999).

[27] C. Barth and C. R. Henry, Phys. Rev. Lett. 91, 196102 (2003). 